\title{
Core-shell III-nitride nanowire heterostructure: negative differential resistance and device application potential
}

\author{
(C) A.M. Mozharov ${ }^{1}$, A.A. Vasiliev ${ }^{1}$, A.D. Bolshakov ${ }^{1}$, G.A. Sapunov ${ }^{1}$, V.V. Fedorov ${ }^{1}$, \\ G.E. Cirlin ${ }^{1,2,3}$, I.S. Mukhin ${ }^{1,2}$
}

${ }^{1}$ St. Petersburg Academic University, Russian Academy of Sciences, 194021 St. Petersburg, Russia

${ }^{2}$ ITMO University,

197101 St. Petersburg, Russia

${ }^{3}$ Institute for Analytical Instrumentation, Russian Academy of Sciences, 190103 St. Petersburg, Russia

E-mail: mozharov@spbau.ru

In this work we have studied volt-ampere characteristics of single core-shell GaN/InGaN GaN nanowire. It was experimentally shown that negative differential resistance effect can be obtained in the studied heterostructure. On the base of numerical calculation results the model describing negative differential resistance phenomenon was proposed. We assume this effect to be related with strong localization of current flow inside the nanowire and emergence of Gunn effect in this area. 